\title{
Revisiting EELS Fine Structure Analysis for Polymers
}

Robert Colby ${ }^{1}$, Robert Williams ${ }^{2}$, Donald Carpenter ${ }^{1}$, Nuria Bagues ${ }^{3}$, David McComb ${ }^{4}$ and Srinivasan Rajagopalan $^{1}$

${ }^{1}$ ExxonMobil Research and Engineering Company, Annandale, New Jersey, United States, ${ }^{2}$ The Ohio State University, Hilliard, Ohio, United States, ${ }^{3}$ The Ohio State University, Columbus, Ohio, United States, ${ }^{4}$ Center for Electron Microscopy and Analysis/ Department of Material Science and Engineering, The Ohio State University, Columbus, Ohio, United States

Electron energy loss spectroscopy (EELS) has been used to analyze polymers and other organic solids for decades, but the fidelity and detail of the near-edge fine structure (ELNES) has historically fallen short of the similar near-edge x-ray absorption spectroscopy (NEXAFS) for light elements like carbon. These shortcomings have variously been attributed to achievable energy resolution, detector signal-to-noise ratio and point spread, and greater beam-induced damage rates for $\sim 100 \mathrm{kV}$ electrons compared to soft $\mathrm{x}$-rays [1]. While NEXAFS analysis of polymers has been used to successfully probe increasingly subtle changes in the functional group chemistry, bonding and orientation of polymers, it typically remains limited to $\sim 40$ $\mathrm{nm}$ resolution and specialized synchrotron endstations.

By contrast, nearly any modern analytical scanning transmission electron microscope (STEM) is capable of producing a sub-nanometer probe. With monochromation, the energy resolutions that can be achieved for STEM are now well below the limits set by core-hole lifetime broadening for polymer-relevant Kedges [2,3]. The development of direct electron detectors for EELS, and the ongoing improvements in detector readout speeds are improving both achievable signal-to-noise and the detector point spread function [4,5]. More recent studies have revised the relative damage rates of polymers for high energy electrons and soft x-rays [6]. Revisiting the use of STEM-EELS for polymer chemistry analysis seems timely.

With this in mind, reference spectra have been collected for a variety of common and model polymers both at room temperature and cooled with liquid nitrogen. Thin sections were prepared by ultramicrotomy at a temperature below the glass transition with a target ratio of thickness to inelastic mean free path of $(t / \lambda)$ of less than 1. Typical references are acquired in STEM by averaging over micron-scale areas with well less than 10 electrons/ $\AA^{2}$. With an energy resolution of $<0.4 \mathrm{eV}$ at full width of half maximum (FWHM) at $200 \mathrm{kV}$, and the conventional CCD-style detector in a Gatan Enfinium, it is possible to obtain EELS spectra that favorably match the characteristic features seen in NEXAFS (Figure 1). Where there are deviations, there are clear indications of the specific beam-induced chemistries responsible. Saturated polymers are in particular sensitive to damage, with clear features of desaturation emerging even at the lowest practical doses afforded by a CCD. Monochromation to $<0.2 \mathrm{eV}$ at $300 \mathrm{kV}$ does offer some improvement in peak-to-background, but is limited by the point spread of a CCD and still falls short of NEXAFS. Further improvements are realized by combining monochromation with a Gatan K2 direct electron detector.

Ultimately, beam-induced damage still imposes that practical limit for spatial resolution polymers with STEM-EELS. Critical doses for polymers are generally only between $1-6$ electrons $/ \AA^{2}$ for mapping "undamaged" polymer spectra. However, with sample cooling and a thin protective coating or backing film, signs of beam-induced chemistry frequently precede significant mass loss or morphology change, meaning that morphology and composition can still be measured. This has been demonstrated using a 
model blend of polycarbonate/poly(styrene-acrylonitrile) (PC/SAN), for which sub-20-nm pixel maps are achievable even with a CCD-based detector when some beam-induced chemistry is tolerable (Figure 2). Given the information available in the carbon K-edge spectra, the practical spatial resolution can be assessed in terms of factors such as the critical dose for the specific chemistry to be measured, the desired energy resolution, and the detector used.

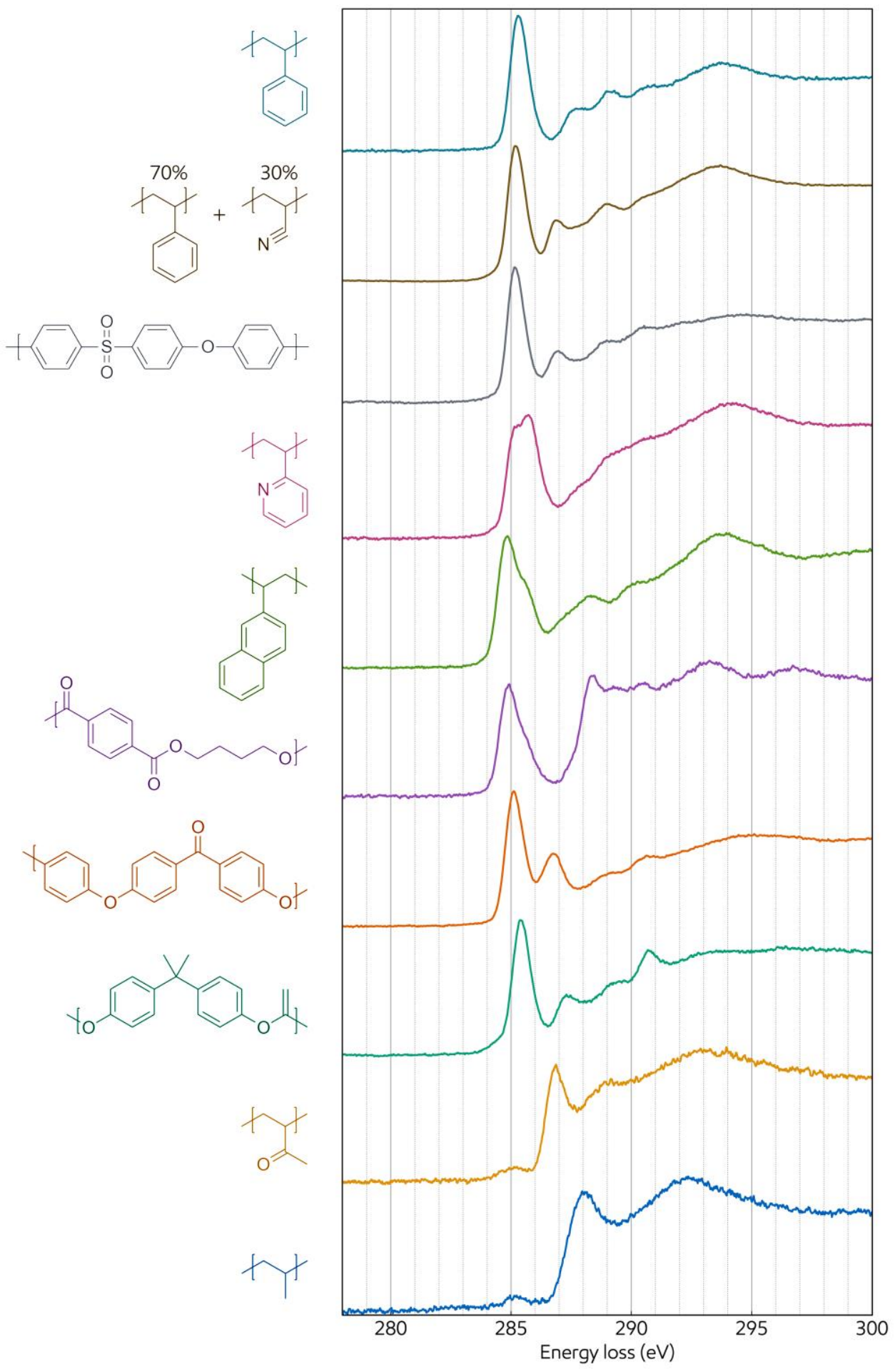


Figure 1. Reference spectra collected for a variety of polymers at $-170^{\circ} \mathrm{C}$, collected at $200 \mathrm{kV}$ with a zero loss FWHM of $\sim 0.4 \mathrm{eV}$ and a fluence of 1-5 electrons/Å2. From top to bottom, the spectra are for polystyrene, poly(styrene-acrylonitrile), poly(ether-sulfone), poly(2-vinylpyridine), poly(2vinylnaphthalene), poly(butylene terephthalate), polyether ether ketone, polycarbonate, poly(vinylmethyl-ketone), and polypropylene. While the energy resolution is well short of NEXAFS, there is good agreement between the primary features observed in each.

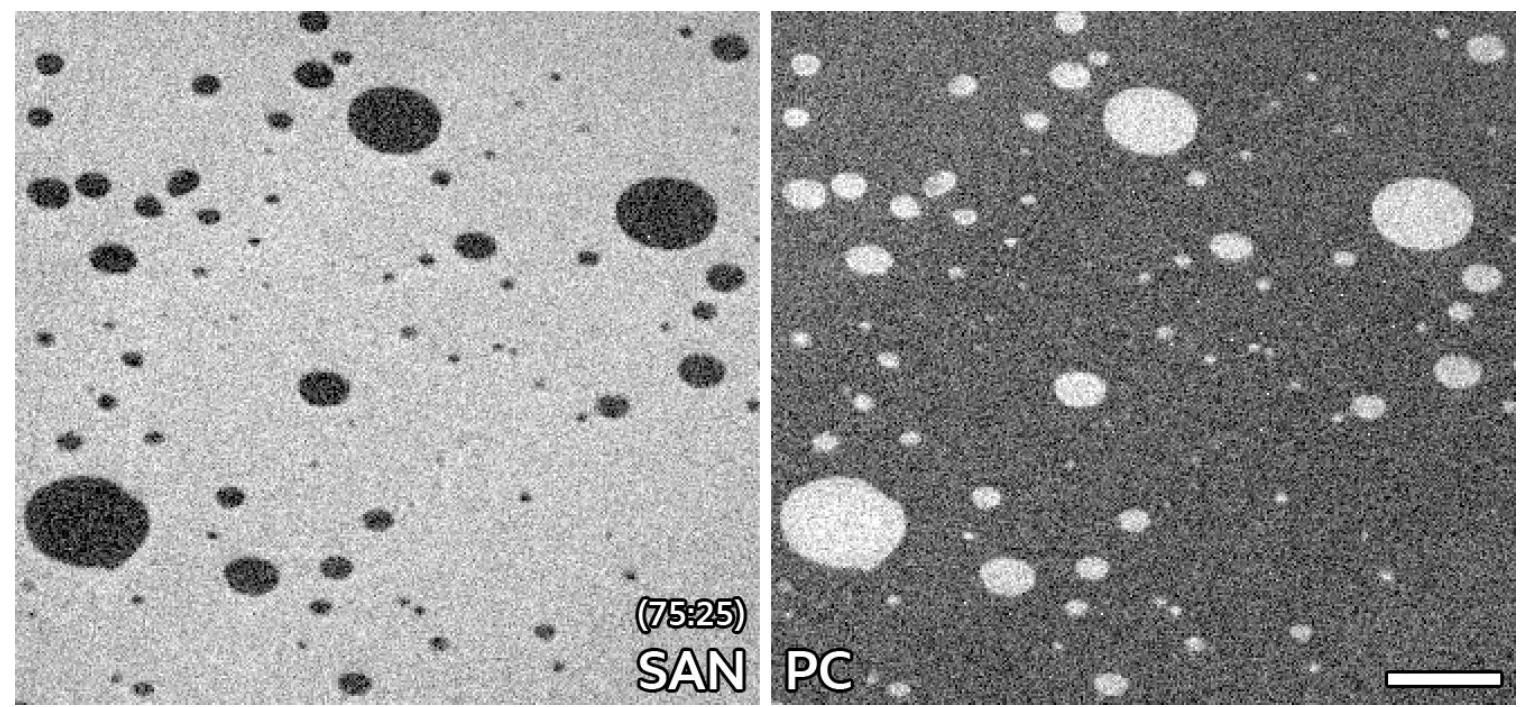

Figure 2. Results of a STEM-EELS map of a model PC/SAN blend acquired at $-170^{\circ} \mathrm{C}$ with 20 -nm pixels, at $200 \mathrm{kV}$, with a zero loss FWHM of $\sim 0.4 \mathrm{eV}$ and a fluence of $\sim 120$ electrons/Å2. The image pair are linear least squares fits to neat PC and SAN (75\% styrene) references acquired under the same conditions, and are sufficient to detect $<50 \mathrm{~nm}$ droplets of PC, as well as the small amount of remaining PC expected in the SAN phase in this system. Scale bar is $1 \mu \mathrm{m}$.

\section{References}

[1] Transmission Electron Energy Loss Spectrometry in Materials Science and The EELS Atlas, 2004, Chapter 12, pages 419-454.

[2] Microscopy, 62, 1, 2013, pages 3-21.

[3] J. Phys.: Conf. Ser. 522, 2014, page 012023.

[4] Microscopy, 67, Supplement 1, 2018, pages i86-i97.

[5] Sci. Rep. 7, 2017, 8243.

[6] J. Phys. Chem. B, 113, 7, 2009, pages 1869-1876. 